ARTIGO ORIGINAL ORIGINAL ARTICLE

\title{
Análise de custo por resposta de adalimumabe, etanercepte, guselcumabe, infliximabe, ixequizumabe, secuquinumabe e ustequinumabe para tratamento de psoríase em placas moderada a grave sob a perspectiva do Sistema de Saúde Suplementar brasileiro
}

\author{
Cost per response analysis of adalimumab, etanercept, \\ guselkumab, infliximab, ixekizumab, secukinumab and \\ ustekinumab for the treatment of moderate to severe plaque \\ psoriasis from the Brazilian private health care system perspective \\ Cyntia Fioratti ${ }^{1}$, Atanacio Valencia-Mendoza ${ }^{2}$, Maria Luiza Rachid', \\ Ricardo Rosim', Marcela Junqueira' \\ DOI: 10.21115/JBES.v10.n3.p226-31
}

\section{Palavras-chave:}

psoríase em placas, custo por resposta, adalimumabe, etanercepte, guselcumabe, infliximabe, ixequizumabe, secuquinumabe, ustequinumabe

\section{Keywords:}

plaque psoriasis, cost per response, adalimumab, etanercept, guselkumab, infliximab, ixekizumab, secukinumab, ustekinumab

\section{RESUMO}

Objetivo: $O$ objetivo deste estudo é avaliar o custo por resposta das terapias biológicas disponíveis no Brasil para o tratamento da psoríase em placas moderada a grave na perspectiva do Sistema de Saúde Suplementar. Métodos: A resposta PASI 90 foi o desfecho avaliado neste estudo. Dados clínicos foram calculados com base na razão de risco de uma metanálise em rede, comparando adalimumabe, etanercepte, infliximabe, ixequizumabe, secuquinumabe e ustequinumabe a guselcumabe, cujo dado foi obtido no estudo clínico. Foram considerados apenas os custos de medicamentos. O caso-base avaliou o custo por resposta do ano de indução do tratamento. Além disso, conduziu-se uma análise de orçamento fixo. Em um cenário alternativo, analisou-se o custo por resposta do ano de manutenção. Uma análise de sensibilidade avaliou incertezas dos dados clínicos. Resultados: 0 menor custo por resposta foi de guselcumabe ( $R \$ 98.643)$, seguido de ixequizumabe (R\$112.549), ustequinumabe ( $R \$ 1$ 124.078), secuquinumabe (R\$160.930), infliximabe ( $R \$ 208.039)$, adalimumabe ( $R \$ 208.686)$ e etanercepte ( $\mathrm{R} \$$ 639.124). Resultados similares foram observados no cenário alternativo, considerando os custos no ano de manutenção. Guselcumabe demonstrou ser a terapia que tratou mais pacientes com sucesso, considerando um cenário de orçamento fixo. Conclusão: 0 presente estudo demonstrou que, na perspectiva do Sistema de Saúde Suplementar brasileiro, guselcumabe possui o menor custo por resposta entre as terapias biológicas para psoríase em placas moderada a grave, além de tratar com sucesso mais pacientes em um cenário de orçamento fixo.

\section{ABSTRACT}

Objective: This study aims to evaluate the cost per response of the biologic therapies available for moderate to severe plaque psoriasis treatment in Brazil from a private payer perspective. Methods: Treatment response evaluated in this study was the achievement of PASI 90. Clinical data was calculated based on the risk ratio of a network meta-analysis comparing adalimumab, etanercept, infliximab, ixekizumab, secukinumab and ustekinumab to guselkumab, which data was extracted from clinical trials. Only drug acquisition costs were considered. Base case analysis

\footnotetext{
Recebido em: 09/11/2018. Aprovado para publicação em: 18/11/2018.

1. Janssen-Cilag Farmacêutica, São Paulo, SP, Brasil.

2. Janssen-Cilag Farmacêutica, México.

Instituição onde o trabalho foi executado: Janssen-Cilag Farmacêutica.

Informações sobre auxílios recebidos sob a forma de financiamento, equipamentos ou medicamentos: 0 projeto fo realizado pela Janssen Brasil.

Autor correspondente: Cyntia Fioratti. Av. Presidente Juscelino Kubitschek, 2041, bloco B, São Paulo, SP, Brasil. CEP: $04543-011$. Telefone: (11) 2788-4728. E-mail: cfioratt@its.jnj.com
} 
evaluated the first year of treatment cost per response. Besides that, a fixed budget analysis was conducted. An alternative scenario analysis considered the maintenance year cost per response. A sensitivity analysis evaluated the clinical data uncertainties. Results: The lowest cost per response was obtained with guselkumab (R\$98.643), followed by ixekizumab (R\$112.549), ustekinumab (R\$124.078), secukinumab (R\$ 160.930), infliximab (R\$ 208.039), adalimumab (R\$ 208.686), and etanercept (R\$ 639.124). Similar results were found in the alternative scenario, with maintenance year costs. Guselkumab demonstrated to be the therapy which successfully treats more patients with a fixed budget. Conclusion: In conclusion, this study demonstrated that, from the Brazilian private payer perspective, guselkumab presents the lowest cost per response among the available biologic therapies for moderate to severe plaque psoriasis, and is able to successfully treat more patients in a limited budget scenario.

\section{Introdução}

A psoríase é uma doença de pele inflamatória crônica, imunomediada, multissistêmica, com comorbidades associadas (Golbari et al., 2018; Sociedade Brasileira de Dermatologia, 2012; National Institute for Health and Clinical Excellence, 2012), que acomete homens e mulheres de todas as idades, porém com picos de incidência aos 20 e 50 anos (Sociedade Brasileira de Dermatologia, 2012). A prevalência da psoríase pode variar, mas estima-se que seja de $2 \%$ a $4 \%$ na população ocidental e de 1,31\% na população brasileira (Golbari et al., 2018; Sociedade Brasileira de Dermatologia, 2012; Romiti et al., 2017). Características genéticas, étnicas, geográficas e ambientais podem influenciar no acometimento pela doença (Sociedade Brasileira de Dermatologia, 2012).

A psoríase em placas é a manifestação mais comum da psoríase e é caracterizada por placas eritematoescamosas, bem delimitadas, ocasionalmente pruriginosas (Consenso $\mathrm{SBD})$, que podem acometer áreas mais restritas ou toda a pele (Sociedade Brasileira de Dermatologia, 2012; Canadian Psoriasis Guidelines Committee, 2009). Além de sintomas como dor e prurido, essa manifestação clínica pode causar debilidade física e isolamento social (Canadian Psoriasis Guidelines Committee, 2009).

Por ser uma doença crônica, o tratamento da psoríase almeja a redução de sintomas e a melhoria na qualidade de vida do paciente (Golbari et al., 2018). A gravidade da doença e a eficácia do tratamento são avaliadas por índices como o de avaliação da área de superfície corporal (BSA), o índice de área e gravidade da psoríase (PASI) e, inclusive, medidas de qualidade de vida (Canadian Psoriasis Guidelines Committee, 2009; Gisondi et al., 2017). O PASI 75, que mensura uma meIhora de pelo menos 75\% das lesões de psoríase em comparação ao início do tratamento ou à medida de PASI anterior, foi por muito tempo o principal desfecho dos estudos clínicos. Entretanto, com o surgimento de medicamentos mais eficazes, o desfecho mais relevante atualmente passou a ser o PASI 90, ou seja, a proporção de pessoas que atingem me-
Ihora de pelo menos 90\% em relação ao início do tratamento (Gisondi et al., 2017).

Existem diversas diretrizes publicadas com algoritmos de tratamento para a psoríase, porém algumas variáveis devem ser consideradas na escolha da conduta clínica, como comorbidades do paciente, gravidade da doença, regiões acometidas, preferência do paciente, entre outras (Golbari et al., 2018; National Institute for Health and Clinical Excellence, 2012; Canadian Psoriasis Guidelines Committee, 2009). O National Institute for Health and Care Excellence (NICE), por exemplo, recomenda para os casos leves as terapias tópicas como primeira linha de tratamento, a fototerapia e os medicamentos sistêmicos não biológicos como segunda linha e os sistêmicos biológicos como terceira linha (National Institute for Health and Clinical Excellence, 2012). Para pacientes com psoríase grave ou extensa (PASI > 10) que não pode ser controlada com terapia tópica e tem significativo impacto em seu bem-estar, a recomendação é iniciar medicamentos sistêmicos não biológicos (National Institute for Health and Clinical Excellence, 2012). O Consenso Brasileiro de Psoríase recomenda que quadros leves sejam tratados com medicações tópicas e quadros moderados a graves sejam inicialmente tratados com fototerapia e, posteriormente, com os sistêmicos tradicionais e biológicos (Sociedade Brasileira de Dermatologia, 2012). Por outro lado, o guia canadense preconiza que não há motivo clínico para restringir medicamentos biológicos para linhas posteriores no tratamento de psoríase moderada a grave e que seus benefícios representam um importante fator de escolha da conduta clínica (Canadian Psoriasis Guidelines Committee, 2009).

A cronicidade da psoríase e a necessidade de tratamento contínuo acarretam uma carga social e econômica para o paciente e para o sistema de saúde (Raho et al., 2012). Constatou-se uma correlação entre o aumento da gravidade da doença e o aumento dos custos diretos e indiretos associados ao tratamento (Feldman et al., 2014). Além disso, estudos mostram que pacientes com comorbidades apresentam taxas de internação e atendimentos ambulatoriais e emer- 
genciais significativamente maiores, incorrendo em maiores custos (Kimball et al., 2011). A introdução da terapia biológica reduz custos diretos em pacientes com psoríase, principalmente ao reduzir o número de diárias hospitalares (Driessen et al., 2010). Diante de um cenário de recursos limitados, o objetivo deste estudo é avaliar o custo por resposta dos medicamentos biológicos aprovados no Brasil para tratamento de psoríase em placas sob a perspectiva do Sistema de Saúde Suplementar (SSS).

\section{Métodos}

O presente estudo avaliou o custo por resposta dos medicamentos adalimumabe, guselcumabe, etanercepte, infliximabe, ixequizumabe, secuquinumabe e ustequinumabe em um horizonte de tempo de 52 semanas. O desfecho clínico avaliado foi a taxa de resposta PASI 90. Devido à ausência de um estudo head-to-head que compare esses tratamentos, utilizaram-se dados de razão de risco de metanálise em rede para comparação indireta com guselcumabe (Tabela 1), cujo dado foi obtido no estudo VOYAGE 1, referente ao desfecho de PASI 90 em 48 semanas, o que corresponde a uma taxa de resposta de 0,76 (Cameron et al., 2018; Blauvelt et al., 2017).

Tabela 1. Razão de risco utilizada para cálculo do PASI 90

\begin{tabular}{lccc}
\hline & $\begin{array}{c}\text { Razão de risco } \\
\text { (guselcumabe } \\
\text { vs. }\end{array}$ & & \\
Tratamento & comparador) & IC 95\% inferior & $\begin{array}{c}\text { IC 95\% } \\
\text { superior }\end{array}$ \\
\hline Ixequizumabe & 1,00 & 0,88 & 1,14 \\
\hline Secuquinumabe & 1,19 & 1,03 & 1,38 \\
\hline Infliximabe & 1,29 & 1,07 & 1,56 \\
\hline Adalimumabe & 1,48 & 1,30 & 1,68 \\
\hline Ustequinumabe & 1,57 & 1,33 & 1,84 \\
\hline Etanercepte & 5,37 & 3,71 & 8,06 \\
\hline
\end{tabular}

Para o custo dos tratamentos, considerou-se apenas a aquisição dos medicamentos, que foi calculada com base no PF18\% da Tabela da Câmara de Regulação do Mercado de Medicamentos (Anvisa, 2018) (Tabela 2) e na posologia aprovada em bula, considerando como referência $70 \mathrm{~kg}$ de peso corpóreo (Tremfya, 2018; Humira, 2018; Stelara, 2018; Enbrel, 2018; Remicade, 2018; Cosentyx, 2018; Taltz, 2018). O caso-base considera o custo de tratamento até a semana 52 do ano de indução e em análise de cenário alternativo utiliza-se o custo do ano de manutenção.

Adicionalmente, analisou-se a efetividade do tratamento em um cenário de orçamento fixo. Calculou-se a quantidade de pacientes que receberiam tratamento com um orçamento de 1 milhão de reais e, com base nos dados clínicos, estimaram-se aqueles que teriam sucesso com cada um dos tratamentos.

Realizou-se uma análise de sensibilidade variando os riscos relativos da metanálise com os valores do intervalo de confiança de 95\% (Tabela 1).

\section{Resultados}

Os resultados de taxa de resposta (PASI 90), do custo na semana 52 do ano de indução e de custo por resposta encontram-se na Tabela 3. Guselcumabe foi o tratamento que apresentou o melhor resultado de custo por resposta entre as terapias avaliadas, seguido de ixequizumabe, ustequinumabe, secuquinumabe, infliximabe, adalimumabe e etanercepte (Figura 1).

O resultado da análise de eficiência com orçamento fixo encontra-se na Figura 2. As barras representam a quantidade de pacientes que recebem cada terapia com um orçamento de 1 milhão de reais. A parcela da barra no eixo positivo representa os pacientes que são tratados com sucesso, nesse caso os que atingem a resposta PASI 90, e a parcela no eixo

Tabela 2. Posologia de cada tratamento e preço lista

\begin{tabular}{|c|c|c|}
\hline Medicamento & Posologia & PF18\% \\
\hline Adalimumabe (40 mg/2 seringas preenchidas) & $\begin{array}{l}\text { Dose inicial de } 80 \text { mg, seguida de } 40 \text { mg em semanas } \\
\text { alternadas, uma semana após a dose inicial. (Humira, 2018) }\end{array}$ & $R \$ 7.683,91$ \\
\hline Guselcumabe (100 mg/1 seringa preenchida) & $\begin{array}{l}100 \text { mg na semana } 0 \text { e na semana 4, seguida de doses } \\
\text { de manutenção a cada } 8 \text { semanas. (Tremfya, 2018) }\end{array}$ & $R \$ 10.750,88$ \\
\hline Etanercepte (50 mg/4 seringas preenchidas) & $\begin{array}{l}50 \text { mg uma vez por semana por até } 12 \text { semanas, } \\
\text { seguida, se necessário, por doses semanais de } \\
50 \text { mg. (Enbrel, 2018) }\end{array}$ & $\mathrm{R} \$ 6.984,66$ \\
\hline Infliximabe (100 mg/1 frasco ampola) & $\begin{array}{l}\text { Infusão intravenosa de } 5 \text { mg/kg, nas semanas 0, } 2 \text { e 6, seguida por } \\
\text { doses de infusões de } 5 \text { mg/kg a cada } 8 \text { semanas. (Remicade, 2018) }\end{array}$ & $R \$ 3.844,88$ \\
\hline Ixequizumabe (80 mg/1 seringa preenchida) & $\begin{array}{l}160 \text { mg (duas injeções de } 80 \text { mg) na semana 0, } \\
\text { seguida por uma injeção de } 80 \text { mg nas semanas 2, 4, 6, 8, } \\
10 \text { e } 12 \text { e, então, } 80 \text { mg a cada } 4 \text { semanas. (Taltz, 2018) }\end{array}$ & $R \$ 5.052,95$ \\
\hline Secuquinumabe (150 mg/2 seringas preenchidas) & $\begin{array}{l}300 \text { mg (duas injeções subcutâneas de } 150 \text { mg), com } \\
\text { administração inicial nas semanas 0, 1, 2, } 3 \text { e 4, seguida por } \\
\text { administrações de manutenção mensal. (Cosentyx, 2018) }\end{array}$ & $\mathrm{R} \$ 6.448,32$ \\
\hline Ustequinumabe (45 mg/1 seringa preenchida) & 45 mg nas semanas 0 e 4 e, depois, a cada 12 semanas. (Stelara, 2018) & $\mathrm{R} \$ 12.058,80$ \\
\hline
\end{tabular}


Tabela 3. Resultados de taxa de resposta PASI 90, do custo de tratamento em 52 semanas do ano de indução e do custo por resposta do caso-base

\begin{tabular}{lccc}
\hline Tratamento & Taxa de resposta (PASI 90) & $\begin{array}{c}\text { Custo na semana 52 do } \\
\text { ano de indução }\end{array}$ & Custo por resposta \\
\hline Etanercepte $(50 \mathrm{mg})$ & 0,14 & $\mathrm{R} \$ 90.800,58$ & $\mathrm{R}$ 639.123,94 \\
\hline Adalimumabe $(40 \mathrm{mg})$ & 0,52 & $\mathrm{R} \$ 107.574,74$ & $\mathrm{R} \$ 208.686,42$ \\
\hline Ustequinumabe $(45 \mathrm{mg})$ & 0,49 & $\mathrm{R} \$ 60.294,00$ & $\mathrm{R} \$ 124.078,33$ \\
\hline Secuquinumabe $(150 \mathrm{mg})$ & 0,64 & $\mathrm{R} \$ 103.173,12$ & $\mathrm{R} \$ 160.929,51$ \\
\hline Ixequizumabe $(80 \mathrm{mg})$ & 0,76 & $\mathrm{R} \$ 85.900,15$ & $\mathrm{R}$ 112.549,22 \\
\hline Infliximabe $(100 \mathrm{mg})$ & 0,59 & $\mathrm{R} \$ 123.036,16$ & $\mathrm{R}$ 208.038,95 \\
\hline Guselcumabe $(100 \mathrm{mg})$ & 0,76 & $\mathrm{R} \$ 75.256,16$ & $\mathrm{R}$ 98.642,54 \\
\hline
\end{tabular}

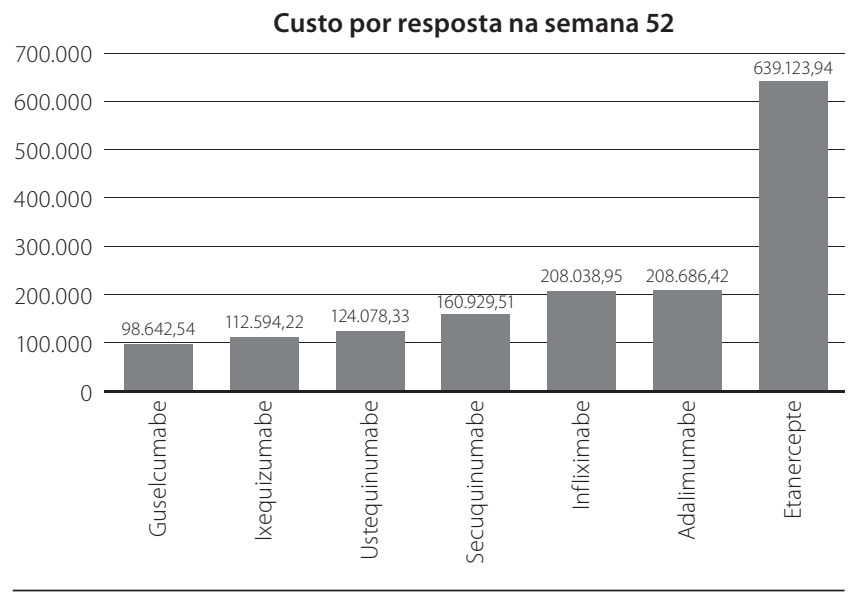

Figura 1. Resultado de custo por resposta PASI 90 em 52 semanas do caso-base.

Eficácia na semana 52

Pacientes tratados com sucesso (PASI $\geq 90$ ) versus pacientes tratados sem sucesso (PASI < 90)

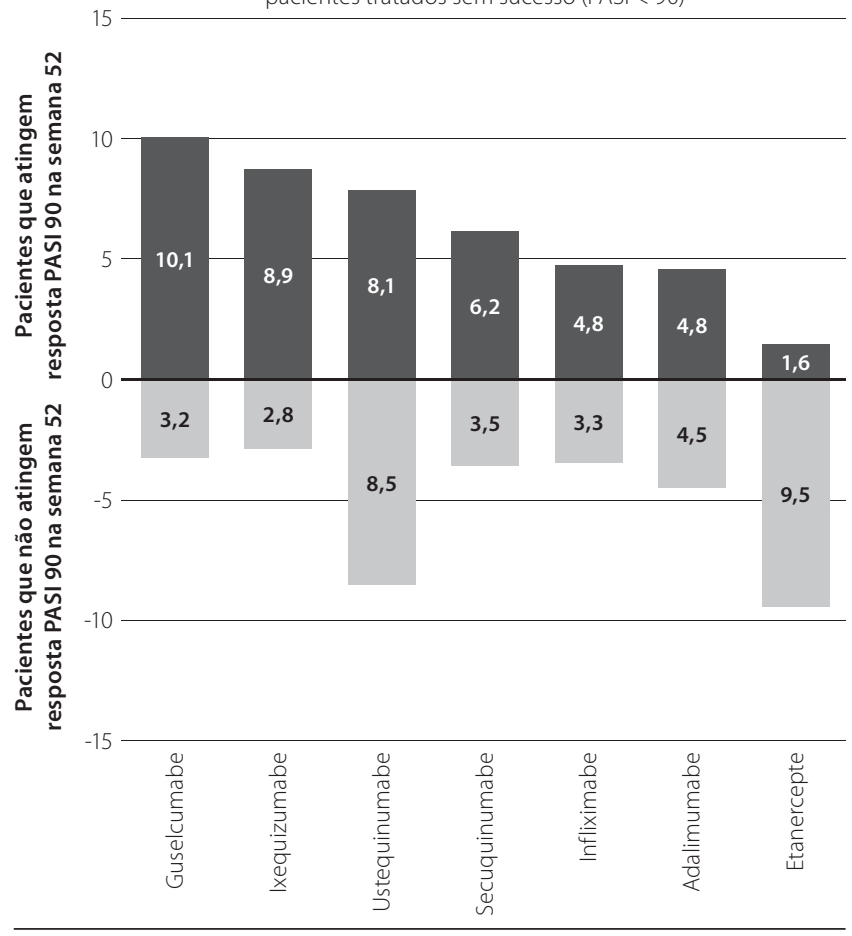

Figura 2. Resultado da análise com orçamento fixo de 1 milhão de reais. negativo representa aqueles que não atingem esse desfecho. Guselcumabe demonstrou ser a terapia capaz de tratar com sucesso mais pacientes no cenário de orçamento fixo, seguido de ixequizumabe, ustequinumabe, secuquinumabe, infliximabe, adalimumabe e etanercepte.

O resultado da análise de cenário alternativo, que considerou o custo de tratamento do ano de manutenção, está representado na Tabela 4 e na Figura 3.

A análise de sensibilidade (Figura 4) constatou que, com exceção de etanercepte, os tratamentos possuem um resultado de custo por resposta com pouca variabilidade quando analisada a incerteza do dado de desfecho clínico.

\section{Discussão}

Entre as terapias biológicas aprovadas no Brasil para o tratamento de psoríase em placas moderada a grave, guselcumabe demonstrou ser a terapia mais custo-efetiva sob a perspectiva do SSS, apresentando o melhor resultado de custo por resposta e com maior número de pacientes tratados com sucesso quando considerado um orçamento fixo.

Tabela 4. Resultados de custo de tratamento no ano de manutenção e custo por resposta do cenário alternativo

\begin{tabular}{|c|c|c|}
\hline Tratamento & $\begin{array}{l}\text { Custo na semana } \\
52 \text { do ano de } \\
\text { manutenção }\end{array}$ & Custo por resposta \\
\hline $\begin{array}{l}\text { Guselcumabe } \\
\text { (100 mg) }\end{array}$ & $R \$ 64.505,28$ & $R \$ 84.550,75$ \\
\hline $\begin{array}{l}\text { Ixequizumabe } \\
\text { (80 mg) }\end{array}$ & $R \$ 65.688,35$ & $\mathrm{R} \$ 86.101,46$ \\
\hline $\begin{array}{l}\text { Ustequinumabe } \\
\text { (45 mg) }\end{array}$ & $R \$ 48.235,20$ & $R \$ 99.262,66$ \\
\hline $\begin{array}{l}\text { Secuquinumabe } \\
\text { (150 mg) }\end{array}$ & $\mathrm{R} \$ 77.379,84$ & $\mathrm{R} \$ 120.697,14$ \\
\hline Infliximabe (100 mg) & $R \$ 92.277,12$ & $\mathrm{R} \$ 156.029,21$ \\
\hline $\begin{array}{l}\text { Adalimumabe } \\
(40 \mathrm{mg})\end{array}$ & $\mathrm{R} \$ 99.890,83$ & $\mathrm{R} \$ 193.780,25$ \\
\hline Etanercepte (50 mg) & $\mathrm{R} \$ 90.800,58$ & $\mathrm{R} \$ 639.123,94$ \\
\hline
\end{tabular}




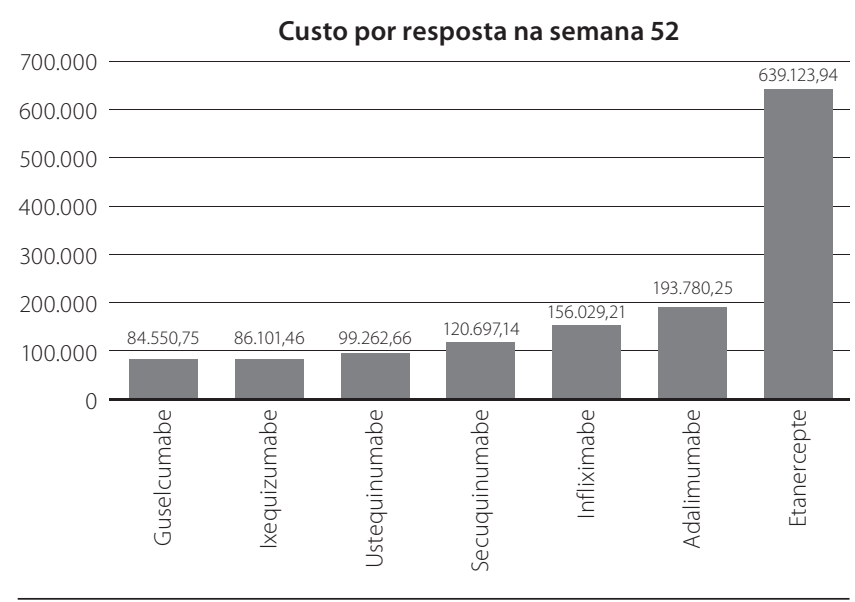

Figura 3. Resultado de custo por resposta PASI 90 em 52 semanas do cenário alternativo.

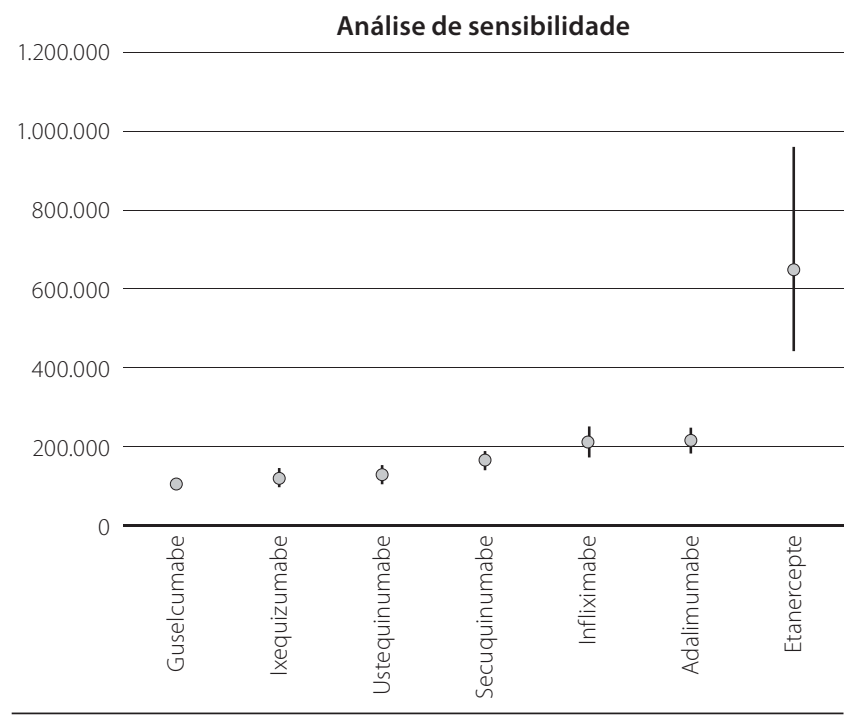

Figura 4. Análise de sensibilidade.

Existem na literatura outros estudos de custo por resposta sob a perspectiva do SSS, porém eles não consideram todas as terapias atualmente aprovadas no Brasil (Scaccabarozzi et al., 2016; Morais \& Ribeiro, 2010). O estudo mais atual analisa apenas adalimumabe, etanercepte, infliximabe e ustequinumabe e, apesar de utilizar o desfecho de PASI 75, os resultados encontrados foram similares aos do presente estudo, no que diz respeito à sequência de terapias com maior custo por resposta, quando considerados os custos no ano de manutenção (Scaccabarozzi et al., 2016).

A análise de cenário alternativo demonstrou que, mesmo quando o custo do ano de manutenção é considerado, o resultado de custo por resposta segue o mesmo padrão. Entretanto, como se observou redução de até $25 \%$ no custo de tratamento no ano de manutenção para algumas terapias, quanto maior a taxa de resposta ao tratamento, menor a necessidade de uma nova terapia e, portanto, menor o gasto com a indução de um novo tratamento.
Uma limitação desse estudo é que os dados de razão de risco utilizados para calcular o desfecho foram obtidos em metanálise em rede que considera dados de PASI 90 do período de indução, que vão no máximo até a semana 16, e o presente estudo considerou esses valores como aproximação para o desfecho na semana 52 (Cameron et al., 2018). Por se tratar de uma doença crônica, a persistência é um fator importante, porém não foi possível ser melhor explorada por essa metodologia. Além disso, medicamentos como ustequinumabe, guselcumabe e secuquinumabe possuem o pico de resposta nas semanas 24, 20 e 16, respectivamente, o que subestima seus respectivos desfechos estimados na semana 52 para o cálculo do custo por resposta, quando se utilizam as razões de risco dessa metanálise em rede (Stelara, 2018; Cosentyx, 2018; Megna et al., 2018).

\section{Conclusão}

Assim, diante de um cenário de recursos limitados, o presente estudo contribui para decisões de gestores do SSS, uma vez que se trata de uma análise que avalia o valor dos tratamentos no âmbito clínico e econômico.

O estudo demonstrou que, na perspectiva do SSS brasileiro, guselcumabe é o tratamento biológico para psoríase em placas moderada a grave com melhor resultado de custo por resposta, seguido de ixequizumabe, ustequinumabe, secuquinumabe, infliximabe, adalimumabe e etanercepte. Além disso, quando analisado um cenário com orçamento fixo, guselcumabe demonstrou ser a terapia que trata com sucesso a maior quantidade de pacientes.

\section{Referências bibliográficas}

Anvisa - Agência Nacional de Vigilância Sanitária. Secretaria Executiva CMED. Lista de preços de medicamentos: preço fábrica e preço máximo ao consumidor. [Atualizado em: 15/06/2018]. Disponível em: http:// portal.anvisa.gov.br/listas-de-precos. Acesso em: 10 jun. 2018.

Blauvelt A, Papp KA, Griffiths CE, Randazzo B, Wasfi Y, Shen YK, et al. Efficacy and safety of guselkumab, an anti-interleukin-23 monoclonal antibody, compared with adalimumab for the continuous treatment of patients with moderate to severe psoriasis: Results from the phase III, doubleblinded, placebo- and active comparator-controlled VOYAGE 1 trial. J Am Acad Dermatol. 2017;76(3):405-17.

Cameron C, Hutton B, Druchok C, McElligott S, Nair S, Schubert A, et al. Importance of assessing and adjusting for cross-study heterogeneity in network meta-analysis: a case study of psoriasis. J Comp Eff Res. 2018;7(11):1037-51.

Canadian Psoriasis Guidelines Committee. Canadian Guidelines for the Management of Plaque Psoriasis. 2009. Disponível em: https://www. dermatology.ca/wp-content/uploads/2012/01/cdnpsoriasisguidelines. pdf. Acesso em: 9 nov. 2018.

Cosentyx: secuquinumabe [bula]. Responsável técnico: Flavia Regina Pegorer. Novartis Biociências S.A.; 2018. 
Driessen RJ, Bisschops LA, Adang EM, Evers AW, Van De Kerkhof PC, De Jong EM. The economic impact of high-need psoriasis in daily clinical practice before and after the introduction of biologics. Br J Dermatol. 2010;162(6):1324-9.

Enbrel: etanercepte [bula]. Responsável técnico: Carolina C. S. Rizoli. Laboratórios Pfizer Ltda.; 2018.

Feldman SR, Burudpakdee C, Gala S, Nanavaty M, Mallya UG. The economic burden of psoriasis: a systematic literature review. Expert Rev Pharmacoecon Outcomes Res. 2014;14(5):685-705.

Gisondi P, Del Giglio M, Girolomoni G. Treatment Approaches to Moderate to Severe Psoriasis. Int J Mol Sci. 2017;18(11).

Golbari NM, Porter ML, Kimball AB. Current guidelines for psoriasis treatment: a work in progress. Cutis. 2018;101(3S):10-2.

Humira: adalimumabe [bula]. Responsável técnico: Carlos E. A. Thomazini. AbbVie Farmacêutica Ltda.; 2018.

Kimball AB, Guérin A, Tsaneva M, Yu AP, Wu EQ, Gupta SR, et al. Economic burden of comorbidities in patients with psoriasis is substantial. J Eur Acad Dermatol Venereol. 2011;25(2):157-63.

Megna M, Balato A, Raimondo A, Balato N. Guselkumab for the treatment of psoriasis. Expert Opin Biol Ther. 2018;18(4):459-68.

Morais AD, Ribeiro F. Cost-effectiveness analysis of biologics in the treatment of moderate to severe psoriasis in the public health care system of Brazil. Value Health. 2010;13(7):A468.
National Institute for Health and Clinical Excellence (NICE). Psoriasis: assessment and management. Geneva; 2012.

Raho G, Koleva DM, Garattini L, Naldi L. The burden of moderate to severe psoriasis: an overview. Pharmacoeconomics. 2012;30(11):1005-13.

Remicade: infliximabe [bula]. Responsável técnico: Marcos R. Pereira. Janssen-Cilag Farmacêutica Ltda.; 2018.

Romiti R, Amone M, Menter A, Miot HA. Prevalence of psoriasis in Brazil - a geographical survey. Int J Dermatol. 2017;56(8):e167-8.

Scaccabarozzi L, Del Rey C, Kim H, Amaral LM, Fernandes RA, Asano E, et al. Análise de custo por resposta dos medicamentos biológicos no tratamento da psoríase moderada a grave sob as perspectivas dos sistemas de saúde público e privado, no Brasil. JBES. 2016;8(2):155-63.

Sociedade Brasileira de Dermatologia. Consenso Brasileiro de Psoríase 2012 Guias de avaliação e tratamento. 2a ed. Rio de Janeiro: SBD; 2012.

Stelara: ustequinumabe [bula]. Responsável técnico: Marcos R. Pereira. Janssen-Cilag Farmacêutica Ltda.; 2018.

Taltz: ixequizumabe [bula]. Responsável técnico: Márcia A. Preda. Eli Lilly do Brasil Ltda.; 2018.

Tremfya: guselcumabe [bula]. Responsável técnico: Marcos R. Pereira. Janssen-Cilag Farmacêutica Ltda.; 2018. 